\title{
Zeros and fixed points of the linear combination of shifts of a meromorphic function
}

\author{
Weiwei Cui ${ }^{1}$, Lianzhong Yang ${ }^{1 *}$ and Xiaoguang Qi $^{2}$
}

\author{
"Correspondence: \\ Izyang@sdu.edu.cn \\ ${ }^{1}$ School of Mathematics, Shandong \\ University, Jinan, Shandong 250100, \\ P.R. China \\ Full list of author information is \\ available at the end of the article
}

\begin{abstract}
Let $f$ be transcendental and meromorphic in the complex plane. In this article, we investigate the existences of zeros and fixed points of the linear combination and quotients of shifts of $f(z)$ when $f(z)$ is of order one. We also prove a result concerning the linear combination which extends a result of Bergweiler and Langley. Some results concerning the order of $f(z)<1$ are also obtained.
\end{abstract}

MSC: 30D35; 39A05

Keywords: entire functions; meromorphic functions; complex difference; fixed points; zeros; shift

\section{Introduction and main results}

In this article, a function is called meromorphic if it is analytic in the whole complex plane except at possible isolated poles. We assume that readers are familiar with the basic results and notations of the Nevanlinna value distribution theory of meromorphic functions (see, e.g., $[1-4])$.

Let $f$ be a transcendental meromorphic function in the plane. The forward differences $\Delta^{n}(f)$ are defined in the standard way ([5], p.52) by $\Delta f=f(z+1)-f(z), \Delta^{n+1}(f)=\Delta^{n} f(z+$ 1) $-\Delta^{n} f(z), n=0,1,2, \ldots$

Recently, many excellent results concerning the Nevanlinna theory for difference operators have been obtained (see, e.g., [6-13]). For example, Halburd and Korhonen [11], respectively Chiang and Feng [7] obtained the difference analogue of logarithmic derivatives lemma. Their results became a starting point of investigating Nevanlinna theory on difference operators and difference equations. Another important result belongs to Bergweiler and Langley. In [6], Bergweiler and Langley firstly investigate the existence of zeros of $\Delta f$ and $\frac{\Delta f}{f}$. The results may be viewed as discrete analogue of the following existence theorem on the zeros of $f^{\prime}$.

Theorem A ([14-16]) Let $f(z)$ be transcendental and meromorphic in the plane with

$$
\varliminf_{r \rightarrow+\infty} \frac{T(r, f)}{r}=0
$$

then $f^{\prime}$ has infinitely many zeros.

(c) 2015 Cui et al. This article is distributed under the terms of the Creative Commons Attribution 4.0 International License (http://creativecommons.org/licenses/by/4.0/), which permits unrestricted use, distribution, and reproduction in any medium, provided you give appropriate credit to the original author(s) and the source, provide a link to the Creative Commons license, and indicate if changes were made. 
Hurwitz theorem implies that under the hypotheses of Theorem A, $f(z+c)-f(z)$ has a zero near $z_{1}$ for all sufficiently small $c \in C \backslash\{0\}$ if $z_{1}$ is a zero of $f^{\prime}$. When $f$ is a transcendental entire function of order less than one, then the first difference $\Delta f$, and by repetition of this argument each difference $\Delta^{n} f$, for $n \geq 1$, is transcendental entire of order less than one and hence has infinitely many zeros. Bergweiler and Langley considered the divided difference case and obtained the following theorem.

Theorem B ([6]) There exists $\delta_{0} \in\left(0, \frac{1}{2}\right)$ with the following property. Let $f$ be a transcendental entire function with order

$$
\sigma(f) \leq \sigma \leq \frac{1}{2}+\delta_{0}<1
$$

then

$$
G(z)=\frac{\Delta f(z)}{f(z)}=\frac{f(z+1)-f(z)}{f(z)}
$$

has infinitely many zeros.

From the proof of Theorem $\mathrm{B}$, it can be seen that $\delta_{0}$ is extremely small, so they conjectured that the conclusion of Theorem B still holds for $\delta(f)<1$. Chen and Shon partly answered this question and obtained the following.

Theorem C ([17]) Let $n \in N$ and $f$ be a transcendental entire function of order $\sigma(f)<1$. Let $c \in C \backslash\{0\}$ and a set $H=\left\{z_{j}\right\}$ consist of all different zeros off $(z)$, satisfying any one of the following two conditions:

(i) at most finitely many zeros $z_{j}, z_{k}$ satisfy $z_{j}-z_{k}=c$;

(ii) $\underline{\lim }_{j \rightarrow+\infty}\left|\frac{z_{j+1}}{z_{j}}\right|=l>1$.

Then

$$
G(z)=\frac{\Delta f(z)}{f(z)}=\frac{f(z+c)-f(z)}{f(z)}
$$

has infinitely many zeros and infinitely many fixed points.

Bergweiler and Langley [6] also obtained the following results.

Theorem D Let $f$ be a function transcendental and meromorphic of lower order $\lambda(f)<\lambda<$ 1 in the plane. Let $c \in C \backslash\{0\}$ be such that at most finitely many poles $z_{j}, z_{k}$ of $f$ satisfy $z_{j}-z_{k}=c$. Then $g(z)=f(z+c)-f(z)$ has infinitely many zeros.

In Theorem D, Bergweiler and Langley considered the existence of zeros of first difference operator when the transcendental meromorphic function is of lower order less than one. Chen and Shon [17] considered the case when the order of $f$ is equal to one. They proved the following results.

Theorem $\mathbf{E}$ Let $c \in C \backslash\{0\}$ and $f$ be a transcendental entire function of order $\sigma(f)=\sigma=1$. If $f(z)$ have infinitely many zeros with the exponent of convergence of zeros $\lambda(f)<1$, then $g(z)=\Delta f(z)=f(z+c)-f(z)$ has infinitely many zeros and infinitely many fixed points. 
In particular, if a set $H=\left\{z_{j}\right\}$ consists of all different zeros off $(z)$, satisfying any one of the following two conditions:

(i) at most finitely many zeros $z_{j}, z_{k}$ satisfy $z_{j}-z_{k}=c$;

(ii) $\underline{\lim }_{j \rightarrow+\infty}\left|\frac{z_{j+1}}{z_{j}}\right|=l>1$,

then

$$
G(z)=\frac{\Delta f(z)}{f(z)}=\frac{f(z+c)-f(z)}{f(z)}
$$

has infinitely many zeros and infinitely many fixed points.

In order to continue to investigate the existence of zeros and fixed points of difference polynomials and their associated difference quotients, we give the linear combination of difference shifts of a meromorphic function as follows:

$$
g(z)=\sum_{j=1}^{n} g_{j}(z) f\left(z+c_{j}\right), \quad G(z)=\left[\sum_{j=1}^{n} g_{j}(z) f\left(z+c_{j}\right)\right] / f(z) .
$$

In fact, $\Delta^{n} f(z)$ is a special case of $g(z)$ since it can be rewritten as the following form:

$$
\Delta^{n} f(z)=\sum_{j=0}^{n} C_{n}^{j}(-1)^{n-j} f(z+j)
$$

By letting the polynomial coefficients of $g(z)$ be constants and $c_{j}$ nonnegative integers, i.e.,

$$
g_{j}(z)=C_{n}^{j-1}(-1)^{n-j+1}, \quad c_{j}=j-1,
$$

for $j=1, \ldots, n+1$, we could find that $g(z)$ and $G(z)$ are general coefficient cases of $\Delta^{n} f(z)$ and $\frac{\Delta^{n} f(z)}{f(z)}$, respectively.

Hence, a natural question arises from Theorem E: What can be said about the existence of zeros and fixed points of $\Delta f$ and $\frac{\Delta f}{f}$, when $f(z)$ is a meromorphic function with order $\sigma(f)=1$ ?

In [18], Cui and Yang considered and gave answers to this question. Now, since we introduce the general form of $\Delta f$ and $\frac{\Delta f}{f}$, the above question acquires its new forms:

Do the conclusions of Theorems B-E still hold for $g(z)$ and $g(z) / f(z)$, when $f(z)$ is a meromorphic function with order $\sigma(f)<1$ or even $\sigma(f) \leq 1$ ? In the present article, we answer this question and obtain the following results.

Theorem 1.1 Let $f$ be a transcendental meromorphic function in the plane with the order $\sigma(f)=\sigma=1$. If $f$ has finitely many poles and infinitely many zeros with the exponent of convergence of zeros $\lambda(f)=\lambda \neq 1$. If the polynomial coefficients $\left\{g_{j}(z)\right\}_{j=1}^{n}$ of $g(z)$ satisfy

$$
\operatorname{deg}\left(g_{l}(z)\right)>\max _{1 \leq j \leq n, j \neq l}\left\{\operatorname{deg}\left(g_{j}(z)\right)\right\}
$$

then $g(z)=\sum_{j=1}^{j=n} g_{j}(z) f\left(z+c_{j}\right)$ has infinitely many zeros and fixed points.

Remark 1.1 By Lemma 2.8 in the following part, we can easily have the following result concerning $\sigma(f)<1$. 
Theorem 1.2 Let $f$ be a transcendental meromorphic function in the plane with the order $\sigma(f)=\sigma<1$. If $f$ has finitely many poles and the polynomial coefficients $\left\{g_{j}(z)\right\}_{j=1}^{n}$ of $g(z)$ satisfy

$$
\operatorname{deg}\left(g_{l}(z)\right)>\max _{1 \leq j \leq n, j \neq l}\left\{\operatorname{deg}\left(g_{j}(z)\right)\right\}
$$

then $g(z)=\sum_{j=1}^{j=n} g_{j}(z) f\left(z+c_{j}\right)$ has infinitely many zeros and fixed points.

Now we consider the existence of zeros and fixed points of $G(z)$. From the proofs of Theorem 1.1 and Theorem 1.2, we notice that the property of $g(z)$, i.e., whether it is transcendental or not, actually determines our conclusions. Therefore, in the following we still first consider the property of $G(z)$ and then investigate zeros and fixed points of $G(z)$ when $f$ is of order equal to or less than one.

Theorem 1.3 Let $G(z)=\frac{g(z)}{f(z)}$ and the polynomial coefficients $\left\{g_{j}(z)\right\}_{j=1}^{n}$ of $g(z)$ satisfy

$$
\operatorname{deg}\left(g_{l}(z)\right)>\max _{1 \leq l \leq n, j \neq l}\left\{\operatorname{deg}\left(g_{j}(z)\right)\right\}, \quad c_{l} \neq 0 .
$$

Then $G(z)$ is transcendental provided $f$ satisfies any one of the following two conditions:

(i) $f$ is transcendental entire and the order of growth of $f$ satisfies

$$
\underline{\lim }_{r \rightarrow+\infty} \frac{T(r, f)}{r}=0
$$

(ii) $f$ is transcendental meromorphic and the order of growth of $f$ satisfies

$$
\underline{\lim }_{r \rightarrow+\infty} \frac{T(r, f)}{r^{\frac{1}{2}}}=0 .
$$

Remark 1.2 In fact, Theorem 1.3 is a generalization of the following last part of Theorem F.

Theorem $\mathbf{F}([6])$ Let $n \in N$ and let $f$ be a transcendental entire function of order $<\frac{1}{2}$, and set

$$
G(z)=\frac{\Delta^{n} f(z)}{f(z)}
$$

If $G$ is transcendental, then $G$ has infinitely many zeros. In particular, iff has order less than $\min \left\{\frac{1}{n}, \frac{1}{2}\right\}$, then $G$ is transcendental and has infinitely many zeros.

In the following, we will investigate the existence of zeros and fixed points of $G(z)$ and obtain some results related to this.

Theorem 1.4 Let $f$ be a function transcendental and meromorphic in the plane with the order $\sigma(f)=\sigma=1$. Iff has finitely many poles and infinitely many zeros with the exponent 
of convergence of zeros $\lambda(f)=\lambda \neq 1$. If the polynomial coefficients $\left\{g_{j}(z)\right\}_{j=1}^{n}$ of $g(z)$ satisfy

$$
\operatorname{deg}\left(g_{l}(z)\right)>\max _{1 \leq j \leq n, j \neq l}\left\{\operatorname{deg}\left(g_{j}(z)\right)\right\}, \quad c_{l} \neq 0,
$$

then $G(z)=\frac{g(z)}{f(z)}$ has infinitely many zeros and fixed points.

The following theorem can be acquired by using Theorem 1.3 and using the same method as in the proof of Theorem 1.2.

Theorem 1.5 Let $f$ be a function transcendental and meromorphic in the plane with the order $\sigma(f)=\sigma<\frac{1}{2}$. If $f(z)$ has only finitely many poles and if the polynomial coefficients $\left\{g_{j}(z)\right\}_{j=1}^{n}$ of $g(z)$ satisfy

$$
\operatorname{deg}\left(g_{l}(z)\right)>\max _{1 \leq j \leq n, j \neq l}\left\{\operatorname{deg}\left(g_{j}(z)\right)\right\}, \quad c_{l} \neq 0,
$$

then $G(z)=\frac{g(z)}{f(z)}$ has infinitely many zeros and fixed points.

In particular, if $f$ is transcendental entire with $\sigma(f)<1$, and the polynomial coefficients $\left\{g_{j}(z)\right\}_{j=1}^{n}$ of $g(z)$ satisfy

$$
\operatorname{deg}\left(g_{l}(z)\right)>\max _{1 \leq j \leq n, j \neq l}\left\{\operatorname{deg}\left(g_{j}(z)\right)\right\}, \quad c_{l} \neq 0,
$$

then $G(z)=\frac{g(z)}{f(z)}$ has infinitely many zeros and fixed points.

\section{Some lemmas}

Lemma 2.1 ([15]) Let $g$ be a function transcendental and meromorphic in the plane of order less than one. Let $h>0$. Then there exists an $\varepsilon$-set $E$ such that

$$
\frac{g^{\prime}(z+c)}{g(z+c)} \rightarrow 0, \quad \frac{g(z+c)}{g(z)} \rightarrow 1,
$$

as $z \rightarrow \infty$ in $C \backslash E$, uniformly in $c$ for $|c| \leq h$. Further, $E$ may be chosen so that for large $z$ not in $E$, the function $g$ has no zeros or poles in $|\zeta-z| \leq h$.

Remark 2.1 ([6]) Following Hayman ([19], pp.75-76), define an $\varepsilon$-set $E$ to be a countable union of discs

$$
E=\bigcup_{j=1}^{\infty} B\left(b_{j}, r_{j}\right)
$$

such that

$$
\lim _{j \rightarrow \infty}\left|b_{j}\right|=\infty, \quad \sum_{j=1}^{\infty} \frac{r_{j}}{\left|b_{j}\right|}<\infty .
$$

Here and henceforth, $B(a, r)$ denotes the open disc of center $a$ and radius $r$, and $S(a, r)$ will denote the corresponding boundary circle. Note that if $E$ is an $\varepsilon$-set, then the set of $r \geq 1$, for which the circle $S(0, r)$ meets $E$, has finite logarithmic measure and hence zero logarithmic density. 
Lemma 2.2 ([4]) Let $f_{j}(z)(j=1, \ldots, n)$ be meromorphicfunctions, $g_{j}(z)(j=1, \ldots, n)$ be entire functions and satisfy:

(i) $\sum_{j=1}^{n} f_{j}(z) e^{g_{j}(z)} \equiv 0$;

(ii) when $1 \leq j<k \leq n, g_{j}(z)-g_{k}(z)$ is not a constant;

(iii) when $1 \leq j \leq n, 1 \leq h<k \leq n$,

$$
T\left(r, f_{j}\right)=o\left(T\left(r, e^{g_{h}-g_{k}}\right)\right) \quad(r \rightarrow \infty, r \notin E),
$$

where $E \subset(1, \infty)$ is of finite linear measure or finite logarithmic measure.

Then $f_{j}(z) \equiv 0(j=1, \ldots, n)$.

Lemma 2.3 ([6]) Letf be a transcendental meromorphic function in the plane of order less than one. Let $h>0$. Then there exists an $\varepsilon$-set $E$ such that

$$
f(z+c)-f(z)=c f^{\prime}(z)(1+o(1))
$$

as $z \rightarrow \infty$ in $C \backslash E$, uniformly in $c$ for $|c| \leq h$.

Lemma 2.4 ([6]) If $f$ is transcendental and meromorphic of order less than one in the plane. Let $n \in N$. Then there exists an $\varepsilon$-set $E_{n}$ such that

$$
\Delta^{n} f(z) \sim f^{(n)}(z)
$$

as $z \rightarrow \infty$ in $C \backslash E_{n}$.

Lemma 2.5 ([20]) Letf be a transcendental meromorphic function with finite order $\sigma(f)=$ $\sigma, H=\left\{\left(k_{1}, j_{1}\right),\left(k_{2}, j_{2}\right), \ldots,\left(k_{q}, j_{q}\right)\right\}$ be a finite set of distinct pairs of integers that satisfy $k_{i}>$ $j_{i} \geq 0$ for $i=1, \ldots, q$, and let $\varepsilon>0$ be a given constant. Then there exists a set $E \subset(1, \infty)$ with finite logarithmic measure such that for all $z$ satisfying $|z| \notin E \cup[0,1]$ and for all $(k, j) \in H$, we have

$$
\left|\frac{f^{(k)}(z)}{f^{(j)}(z)}\right| \leq|z|^{(k-j)(\sigma-1+\varepsilon)}
$$

Lemma 2.6 ([7]) Let $\eta_{1}, \eta_{2}$ be two arbitrary complex numbers and let $f(z)$ be a meromorphic function of finite order $\sigma$. Let $\varepsilon>0$ be given, then there exists a subset $E \subset R$ with finite logarithmic measure such that for all $r \notin E \cup[0,1]$, we have

$$
\exp \left(-r^{\sigma-1+\varepsilon}\right) \leq\left|\frac{f\left(z+\eta_{1}\right)}{f\left(z+\eta_{2}\right)}\right| \leq \exp \left(r^{\sigma-1+\varepsilon}\right)
$$

The following Lemma 2.7 can be easily obtained from Chiang and Feng (Theorem 9.4 [7]), here we omit its proof.

Lemma 2.7 $\operatorname{Let}_{1}(z), \ldots, g_{n}(z)$ be polynomials such that there exists an integer $k, 1 \leq k \leq n$, so that

$$
\operatorname{deg}\left(g_{k}(z)\right)>\max _{1 \leq j \leq n, j \neq k}\left\{\operatorname{deg}\left(g_{j}(z)\right)\right\}
$$


holds. Suppose that $f(z)$ is a meromorphic solution to

$$
g_{n}(z) y\left(z+c_{n}\right)+\cdots+g_{1}(z) y\left(z+c_{1}\right)=0,
$$

where $c_{j}, j=1, \ldots, n$, are complex numbers of which at least two are distinct. Then $\sigma(f) \geq 1$.

Lemma 2.8 Let $f(z)$ be a transcendental meromorphic function satisfying $\sigma(f)=\sigma<1$, and $\left\{g_{j}(z)\right\}$ be polynomials. If there exists an integer $l, 1 \leq l \leq n$, such that

$$
\operatorname{deg}\left(g_{l}(z)\right)>\max _{1 \leq j \leq n, j \neq l}\left\{\operatorname{deg}\left(g_{j}(z)\right)\right\}
$$

holds, then $g(z)$ is transcendental and meromorphic.

Proof Suppose that $g(z)$ is not transcendental. Then we divide our proof into two cases.

Case 1. If $g(z) \equiv 0$, that is,

$$
g_{n}(z) f\left(z+c_{n}\right)+\cdots+g_{1}(z) f\left(z+c_{1}\right) \equiv 0 .
$$

Since

$$
\operatorname{deg}\left(g_{l}(z)\right)>\max _{1 \leq j \leq n, j \neq l}\left\{\operatorname{deg}\left(g_{j}(z)\right)\right\}
$$

holds, and by using Lemma 2.7, we can get that

$$
\sigma(f) \geq 1
$$

Clearly, this contradicts with $\sigma(f)<1$. Hence $g(z) \not \equiv 0$.

Case 2. If $g(z)$ is rational, without loss of generality, we may suppose that $g(z)$ is a nonzero polynomial.

By Lemma 2.1 and $\sigma(f)<1$, we know that there exists an $\varepsilon$-set $E$ such that

$$
f\left(z+c_{j}\right)=f(z)(1+o(1))
$$

as $z \rightarrow \infty$ in $C \backslash E$. Therefore,

$$
g(z)=f(z)\left[g_{1}(z)(1+o(1))+\cdots+g_{n}(z)(1+o(1))\right] .
$$

Since (2.1) holds, we know that

$$
g_{1}(z)+\cdots+g_{n}(z) \not \equiv 0
$$

So, by (2.3) and (2.4), we obtain that

$$
f(z)=\frac{g(z)}{g_{1}(z)(1+o(1))+\cdots+g_{n}(z)(1+o(1))}
$$

as $z \rightarrow \infty$ in $C \backslash E$. The following proof is standard, but a proof will be given for completeness. 
Set $H=\{r:|z|>1, z \in C \backslash E\}$. Thus by Remark 2.1, we see that $H \subset(1,+\infty)$ is of finite logarithmic measure. Since $g(z), g_{j}(z), j=1, \ldots, n$, are polynomials, by $(2.5)$ we get that

$$
\begin{aligned}
T(r, f) & \leq T(r, g)+T\left(r, g_{1}(z)(1+o(1))+\cdots+g_{n}(z)(1+o(1))\right)+O(1) \\
& \leq T(r, g)+\sum_{j=1}^{n} T\left(r, g_{j}\right)+O(1), \quad r \notin H .
\end{aligned}
$$

Thus, for all sufficient large $r$,

$$
T(r, f)=O(\log r)
$$

which contradicts with the fact that $f$ is transcendental.

Hence, $g(z)$ is transcendental.

Lemma $2.9([16,21])$ Iff is transcendental entire satisfying $\underline{\lim }_{r \rightarrow+\infty} \frac{T(r, f)}{r}=0$, or transcendental meromorphic with $\underline{\lim }_{r \rightarrow+\infty} \frac{T(r, f)}{r^{\frac{1}{2}}}=0$, then $\frac{f^{\prime}}{f}$ has infinitely many zeros.

\section{Proofs of Theorem 1.1 and Theorem 1.2}

Proof of Theorem 1.1 According to the condition, suppose that $\left\{b_{v}\right\}, v=1, \ldots, n$, are poles of $f(z)$ with multiplicity $\tau_{v}$, and we let

$$
p(z)=\prod_{v=1}^{n}\left(z-b_{v}\right)^{\tau_{v}}
$$

Then $p(z) f(z)$ is entire and transcendental.

Set $F(z)=p(z) f(z)$. Then, by the Hadamard factorization theorem and $\lambda(f)<\sigma(f)$, we have $F(z)=h(z) e^{a z+b}$, where $a \neq 0$ and $b$ are constants, $h(z)$ is an entire function satisfying $\sigma(h)=\lambda(h)=\lambda(f)<\sigma(f)=1$.

Since $g(z)=\sum_{j=1}^{n} g_{j}(z) f\left(z+c_{j}\right)$ and $F(z)=p(z) f(z)=h(z) e^{a z+b}$, we know that

$$
\begin{aligned}
g(z) & =\sum_{j=1}^{n} g_{j}(z) \frac{h\left(z+c_{j}\right)}{p\left(z+c_{j}\right)} e^{a z+b+a c_{j}} \\
& =\left(\sum_{j=1}^{n} g_{j}(z) \frac{h\left(z+c_{j}\right)}{p\left(z+c_{j}\right)} e^{a c_{j}}\right) e^{a z+b} .
\end{aligned}
$$

Note that $\sigma(h)<1$, by Lemma 2.1, there exists an $\varepsilon$-set $E$ such that

$$
h(z+1) \sim h(z)
$$

as $z \rightarrow \infty$ in $C \backslash E$. Thus

$$
g(z) \sim\left(\sum_{j=1}^{n} g_{j}(z) \frac{1}{p\left(z+c_{j}\right)} e^{a c_{j}}\right) h(z) e^{a z+b} .
$$


Since

$$
\operatorname{deg}\left(g_{l}(z)\right)>\max _{1 \leq j \leq n, j \neq l}\left\{\operatorname{deg}\left(g_{j}(z)\right)\right\}
$$

holds, we know that

$$
\sum_{j=1}^{n} g_{j}(z) \frac{1}{p\left(z+c_{j}\right)} e^{a c_{j}} \not \equiv 0
$$

If $g(z)$ has only finitely many zeros, then there exists a nonzero rational function $R(z)$ such that

$$
\sum_{j=1}^{n} g_{j}(z) \frac{h\left(z+c_{j}\right)}{p\left(z+c_{j}\right)} e^{a c_{j}}=R(z) .
$$

By Lemma 2.8 and $\sigma(h)<1$, we know that (3.1) is impossible. Hence $g(z)$ has infinitely many zeros.

Now we prove that $g(z)=\sum_{j=1}^{n} g_{j}(z) f\left(z+c_{j}\right)$ has infinitely many fixed points. Set

$$
g^{*}(z)=g(z)-z
$$

Thus we only need to prove that $g^{*}(z)$ has infinitely many zeros.

If $g^{*}(z)$ has only finitely many zeros, then we have

$$
g^{*}(z)=R^{*}(z) e^{d z+\alpha}
$$

where $R^{*}(z)$ is a rational function, $d \neq 0$ and $\alpha$ are constants. Without loss of generality, we may suppose that $\alpha=b$. In fact, if $\alpha \neq b$, then $R^{*}(z) e^{d z+\alpha}=e^{\alpha-b} R^{*}(z) e^{d z+b}$, and $e^{\alpha-b} R^{*}(z)$ is also a rational function.

So,

$$
R^{*}(z) e^{d z+b}=h_{0}(z) e^{a z+b}-z
$$

where $h_{0}(z)=\sum_{j=1}^{n} g_{j}(z) \frac{h\left(z+c_{j}\right)}{p\left(z+c_{j}\right)} e^{a c_{j}}$. Since

$$
\operatorname{deg}\left(g_{l}(z)\right)>\max _{1 \leq j \leq n, j \neq l}\left\{\operatorname{deg}\left(g_{j}(z)\right)\right\}
$$

and $\sigma(h)<1$, by Lemma 2.8, we know that $h_{0}(z)$ is transcendental, and $\sigma\left(h_{0}\right)<1$.

We affirm that $a=d$.

If $a \neq d$, then

$$
R^{*}(z) e^{d z+b}-h_{0}(z) e^{a z+b}+z e^{0}=0
$$

This satisfies conditions of Lemma 2.2. Thus, by Lemma 2.2 , we have that $R^{*}(z) \equiv h_{0}(z) \equiv$ $z \equiv 0$, this is a contradiction. Hence $a=d$. 
So,

$$
\left[R^{*}(z)-h_{0}(z)\right] e^{a z+b}=z
$$

again by Lemma 2.2, we get

$$
R^{*}(z)-h_{0}(z) \equiv 0, \quad z \equiv 0 .
$$

This also is a contradiction.

Hence, $g(z)$ has infinitely many fixed points.

Proof of Theorem 1.2 According to Lemma 2.8, we know that $g(z)$ is transcendental under conditions of Theorem 1.2 and the order $\sigma(g)<1$, which means $g(z)$ has either infinitely many zeros or infinitely many poles. Since $f(z)$ has only finitely many poles, i.e., $g(z)$ has finitely many poles. Hence $g(z)$ must have infinitely many zeros.

The same discussion also holds when we consider the fixed points of $g(z)$. Hence, $g(z)$ has infinitely many fixed points.

We complete our proofs of Theorem 1.1 and Theorem 1.2.

\section{Proofs of Theorem 1.3 and Theorem 1.4}

Proof of Theorem 1.3 First we prove that $G(z)$ is transcendental. Suppose that $G$ is rational. Then we obtain that

$$
\sum_{j=1}^{n} g_{j}(z)\left[\frac{f\left(z+c_{j}\right)-f(z)}{f(z)}\right]=R_{1}(z)-\sum_{j=1}^{n} g_{j}(z)
$$

where $R_{1}(z)$ is rational.

According to Lemma 2.3, we know that

$$
f\left(z+c_{j}\right)-f(z)=c_{j} f^{\prime}(z)(1+o(1))
$$

as $z \rightarrow \infty$ in $C \backslash E$. So we get

$$
\sum_{j=1}^{n} c_{j} g_{j}(z) \frac{f^{\prime}(z)}{f(z)}(1+o(1))=R_{1}(z)-\sum_{j=1}^{n} g_{j}(z)
$$

Since $c_{l} \neq 0$ and

$$
\operatorname{deg}\left(g_{l}(z)\right)>\max _{1 \leq j \leq n, j \neq l}\left\{\operatorname{deg}\left(g_{j}(z)\right)\right\}
$$

we know that

$$
\sum_{j=1}^{n} c_{j} g_{j}(z) \not \equiv 0
$$


So,

$$
\frac{f^{\prime}(z)}{f(z)}=\frac{R_{1}(z)-\sum_{j=1}^{n} g_{j}(z)}{\sum_{j=1}^{n} c_{j} g_{j}(z)(1+o(1))} .
$$

Since $g_{j}(z)$ are polynomials. Hence the right-hand side of (4.2) is rational. We can easily get a contradiction from the two sides of (4.2) since condition (i) or (ii) separately guarantees that the left-hand side $\frac{f^{\prime}(z)}{f(z)}$ has infinitely many zeros by Lemma 2.9. Therefore, $G(z)$ is transcendental. We complete the proof of Theorem 1.3.

Proof of Theorem 1.4 Now we prove that $G(z)$ has infinitely many zeros. We continue to use symbols in the proofs of Theorem 1.1 and Theorem 1.2.

Since

$$
\begin{aligned}
G(z) & =\frac{g(z)}{f(z)} \\
& =\frac{\left(\sum_{j=1}^{n} g_{j}(z) \frac{h\left(z+c_{j}\right)}{p\left(z+c_{j}\right)} e^{a c_{j}}\right) e^{a z+b}}{\frac{h(z)}{p(z)} e^{a z+b}} \\
& =\sum_{j=1}^{n} g_{j}(z) \frac{p(z) e^{a c_{j}}}{p\left(z+c_{j}\right)}\left[\frac{h\left(z+c_{j}\right)}{h(z)}\right] \\
& =\sum_{j=1}^{n} g_{j}(z) \frac{p(z) e^{a c_{j}}}{p\left(z+c_{j}\right)} \frac{\Delta_{j} h(z)}{h(z)}+\sum_{j=1}^{n} g_{j}(z) \frac{p(z) e^{a c_{j}}}{p\left(z+c_{j}\right)} \\
& =G_{0}(z)+g_{0}(z),
\end{aligned}
$$

where

$$
g_{0}(z)=\sum_{j=1}^{n} g_{j}(z) \frac{p(z) e^{a c_{j}}}{p\left(z+c_{j}\right)}
$$

is rational, $\Delta_{j} h(z)=h\left(z+c_{j}\right)-h(z)$ and

$$
G_{0}(z)=\sum_{j=1}^{n} g_{j}(z) \frac{p(z) e^{a c_{j}}}{p\left(z+c_{j}\right)} \frac{\Delta_{j} h(z)}{h(z)} .
$$

From Theorem 1.3, since $h(z)$ is transcendental and entire with $\sigma(h)<1$, we know that $G_{0}(z)$ is transcendental and $\sigma\left(G_{0}\right) \leq \sigma(h)<1$, and by Lemma 2.3 and Lemma 2.4, we also know that there exists an $\varepsilon$-set $E$ such that

$$
G_{0}(z) \sim \sum_{j=1}^{n} g_{j}(z) \frac{p(z) e^{a c_{j}}}{p\left(z+c_{j}\right)} \frac{c_{j} h^{\prime}(z)}{h(z)} \sim g_{0}(z) \frac{c_{l} h^{\prime}(z)}{h(z)}
$$

as $z \rightarrow \infty$ in $C \backslash E$, where $E$ contains all zeros and poles of $G_{0}(z)$. Hence, there exists a subset $F_{1} \subset(1, \infty)$ of finite logarithmic measure such that for large $|z|=r$ not in $F_{1}, z \notin E$ and

$$
G(z) \sim g_{0}(z) \frac{c_{l} h^{\prime}(z)}{h(z)}+g_{0}(z) .
$$


We could find a meromorphic function $q(z)$ such that $c_{l} \frac{h^{\prime}}{h}=\frac{q^{\prime}}{q}$. In fact, by integrating both sides of $c_{l} \frac{h^{\prime}}{h}=\frac{q^{\prime}}{q}$, we obtain that $q(z)=A h(z)^{c_{l}}$, where $A$ is a nonzero constant. Since $\sigma(h)<$ 1 , we know that $q(z)$ is of finite order $<1$ and transcendental. Hence, by Lemma 2.5, there exists a set $F_{2} \subset(1, \infty)$ of finite logarithmic measure for any given $\varepsilon(0<2 \varepsilon<1-\sigma(q))$, such that for large $|z|=r$ not in $F_{2}$,

$$
\left|g_{0}(z) \frac{c_{l} h^{\prime}(z)}{h(z)}\right|=\left|g_{0}(z) \frac{q^{\prime}(z)}{q(z)}\right| \leq\left|g_{0}(z)\right| \cdot|z|^{\sigma(q)-1+\varepsilon} .
$$

Define an $\varepsilon$-set $E^{*}$ which consists of all zeros and poles of $G(z)$, then there exists a set $F_{3} \subset(1, \infty)$ of finite logarithmic measure such that if $z \in E^{*}$, then $|z|=r \in F_{3}$.

Thus, by (4.3) and (4.4), we see that for large $|z|=r \notin[0,1] \cup F_{1} \cup F_{2} \cup F_{3}, G(z)$ has no zeros and poles on $|z|=r$ and

$$
\begin{aligned}
\left|G(z)-g_{0}(z)\right| & =\left|G_{0}(z)\right| \\
& =\left|g_{0}(z) \frac{c_{l} h^{\prime}(z)}{h(z)}(1+o(1))\right| \leq\left|g_{0}(z)\right| \cdot|z|^{-\varepsilon}<|G(z)|+\left|-g_{0}(z)\right|
\end{aligned}
$$

holds on $|z|=r$.

According to the Rouché theorem, we obtain that

$$
n\left(r, \frac{1}{G(z)}\right)-n(r, G(z))=n\left(r, \frac{1}{g_{0}(z)}\right)-n\left(r, g_{0}(z)\right)=\operatorname{deg}\left(g_{l}\right) \geq 1
$$

Since $G(z)$ is transcendental and $\sigma(G(z))<1$, we know that at least one of $n\left(r, \frac{1}{G(z)}\right) \rightarrow \infty$ and $n(r, G(z)) \rightarrow \infty$ (as $r \rightarrow \infty$ ) holds. By (4.6), $G(z)$ has either finitely many zeros and poles or infinitely many zeros and poles. Thus, we obtain that

$$
n(r, G(z)) \rightarrow \infty
$$

and

$$
n\left(r, \frac{1}{G(z)}\right) \rightarrow \infty
$$

That is to say, $G(z)$ must have infinitely many zeros.

Let $G^{*}(z)=G(z)-z$, we need to consider zeros of $G^{*}(z)$ in order to estimate the fixed points of $G(z)$. Since $G(z)=G_{0}(z)+g_{0}(z)$, we obtain the following inequality by using the above methods and notations:

$$
G_{*}(z)-g_{0}(z)=G_{0}(z)-z \sim g_{0}(z) \frac{c_{l} h^{\prime}(z)}{h(z)}-z=g_{0}(z) \frac{q^{\prime}(z)}{q(z)}-z .
$$

We could let $g_{0}(z) \frac{q^{\prime}(z)}{q(z)}-z=g_{0}(z) \frac{m^{\prime}(z)}{m(z)}$ since by integrating we obtain that $m(z)=\frac{q}{B \exp \int \frac{z}{g_{0}} d z}$, where $B$ is a nonzero constant. $m(z)$ is of finite order because $q(z)$ is of finite order. And we let $r(z)=\int \frac{z}{g_{0}} d z$, then $r^{\prime}(z)=\frac{z}{g_{0}}$, which means $\exp \int \frac{z}{g_{0}} d z$ is also of finite order. Therefore, we can obtain that

$$
G_{*}(z)-g_{0}(z) \sim g_{0}(z) \frac{m^{\prime}(z)}{m(z)} .
$$


The following proof is similar to the above. We can get a similar equation to that of (4.6) by applying the Rouché theorem. At last, we get our conclusion that $G(z)$ has infinitely many fixed points.

Theorem 1.4 follows.

\section{Proof of Theorem 1.5}

When $f(z)$ is meromorphic with $\sigma(f)<\frac{1}{2}$, since

$$
\operatorname{deg}\left(g_{l}(z)\right)>\max _{1 \leq j \leq n, j \neq l}\left\{\operatorname{deg}\left(g_{j}(z)\right)\right\}, \quad c_{l} \neq 0
$$

by Theorem 1.3 we could immediately find that $G(z)$ is transcendental and the order of $G(z)$ is less than $\frac{1}{2}$, which means $G(z)$ has either infinitely many zeros or infinitely many poles. In what follows, we could obtain

$$
n\left(r, \frac{1}{m(z)}\right)-n(r, m(z))=n\left(r, \frac{1}{g(z)}\right)-n(r, g(z))=\operatorname{deg}\left(g_{l}\right) \geq 1
$$

by using the same method as in the proof of Theorem 1.4, where $m(z)$ represents $G(z)$ or $G(z)-z$. Therefore, $G(z)$ must have infinitely many zeros and fixed points.

When $f(z)$ is transcendental and entire with $\sigma(f)<1$, we can also obtain the same conclusion by the same discussion as above. Theorem 1.5 follows.

\section{Competing interests}

The authors declare that they have no competing interests.

\section{Authors' contributions}

Cui and Yang completed the main part of the article, Qi corrected the main theorems. All authors read and approved the final manuscript.

\section{Author details}

${ }^{1}$ School of Mathematics, Shandong University, Jinan, Shandong 250100, P.R. China. ${ }^{2}$ School of Mathematics, University of Jinan, Jinan, Shandong 250022, P.R. China.

\section{Acknowledgements}

The authors thank the referee for his/her valuable suggestions to improve the present article. The authors also thank Jun Wang for valuable suggestions and discussions. This work was supported by the NNSF of China (No. 11171013 \& No. 11371225). The third author is also supported by the NNSF of China (No. 11301220), the Tianyuan Fund for Mathematics (No. 11226094), the NSF of Shandong Province, China (No. ZR2012AQ020) and the Fund of Doctoral Program Research of University of Jinan (XBS1211).

Received: 14 January 2015 Accepted: 2 September 2015 Published online: 09 September 2015

\section{References}

1. Hayman, WK: Meromorphic Functions. Clarendon, Oxford (1964)

2. Laine, I: Nevanlinna Theory and Complex Differential Equations. de Gruyter, Berlin (1993)

3. Yang, L: Value Distribution Theory and New Research. Science Press, Beijing (1982) (in Chinese)

4. Yi, HX, Yang, CC: The Uniqueness Theory of Meromorphic Functions. Science Press, Beijing (1995) (in Chinese)

5. Whittaker, JM: Interpolatory Function Theory. Cambridge Tracts in Math and Math Phys., vol. 33. Cambridge University Press, Cambridge (1935)

6. Bergweiler, W, Langley, JK: Zeros of differences of meromorphic functions. Math. Proc. Camb. Philos. Soc. 142 133-147 (2007)

7. Chiang, YM, Feng, SJ: On the Nevanlinna characteristic of $f(z+\eta)$ and difference equations in the complex plane. Ramanujan J. 16, 105-129 (2008)

8. Chiang, YM, Feng, SJ: On the growth of logarithmic differences, difference quotients and logarithmic derivatives of meromorphic functions. Trans. Amer. Math. Soc. 361(7), 3767-3791 (2009)

9. Ding, J, Yang, LZ: Value distribution of difference operator on meromorphic functions. Bull. Korean Math. Soc. 51(5), 1291-1297 (2014)

10. Halburd, RG, Korhonen, R: Nevanlinna theory for the difference operator. Ann. Acad. Sci. Fenn. Math. 31, 463-478 (2006) 
11. Halburd, RG, Korhonen, R: Difference analogue of the lemma on the logarithmic derivative with applications to difference equations. J. Math. Anal. Appl. 314, 477-487 (2006)

12. Agarwal, P, Choi, J, Paris, RB: Extended Riemann-Liouville fractional derivative operator and its applications. J. Nonlinear Sci. Appl. 8, 451-466 (2015)

13. Ayad, A, Fares, A, Ayyad, Y: An algorithm for solving zero-dimensional parametric systems of polynomial homogeneous equations. J. Nonlinear Sci. Appl. 5(6), 426-438 (2012) (special issue)

14. Bergweiler, W, Eremenko, A: On the singularities of the inverse to a meromorphic function of finite order. Rev. Mat. Iberoam. 11, 355-373 (1995)

15. Eremenko, A, Langley, JK, Rossi, J: On the zeros of meromorphic functions of the form $\sum_{k=1}^{\infty} \frac{a_{k}}{z-z_{k}}$. J. Anal. Math. 62, 271-286 (1994)

16. Hinchliffe, JD: The Bergweiler-Eremenko theorem for the finite lower order. Results Math. 43, 121-128 (2003)

17. Chen, ZX, Shon, KH: On zeros and fixed points of differences of meromorphic functions. J. Math. Anal. Appl. 344, 373-383 (2008)

18. Cui, WW, Yang, LZ: Zeros and fixed points of difference operators of meromorphic functions. Acta Math. Sci. 33B(3), 773-780 (2013)

19. Hayman, WK: Slowly growing integral and subharmonic functions. Comment. Math. Helv. 34, 75-84 (1960)

20. Gundersen, G: Estimates for the logarithmic derivative of a meromorphic function, plus similar estimates. J. Lond. Math. Soc. 37(2), 88-104 (1988)

21. Clunie, J, Eremenko, A, Rossi, J: On equilibrium points of logarithmic and Newtonian potential. J. Lond. Math. Soc 47(2), 309-320 (1993)

\section{Submit your manuscript to a SpringerOpen ${ }^{\circ}$ journal and benefit from:}

- Convenient online submission

Rigorous peer review

- Immediate publication on acceptance

- Open access: articles freely available online

- High visibility within the field

- Retaining the copyright to your article 